\title{
Modelagem da pandemia Covid-19 como objeto complexo (notas samajianas)
}

NAOMAR DE ALMEIDA FILHO ${ }^{I}$

\section{Introdução}

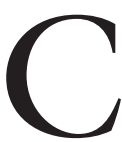

OM RIGOR epistemológico, como modelar a pandemia Covid-19, respeitando sua complexidade, totalidade e singularidade?

Uma pandemia é um evento singular, emergente, totalizado e complexo, tal como furacões, tsunamis, guerras, supernovas e outros fenômenos do mundo e da vida. Alguns autores contemporâneos nomeiam esses fenômenos como eventos críticos (Das, 1996), outros preferem a designação de objetos complexos (Elliot, 2006). Pandemias não se reduzem à emergência de um novo patógeno no bioma terrestre, Sars-Cov-2, nem aos sinais e sintomas inicialmente desconhecidos de uma nova entidade mórbida batizada de Covid-19, nem a curvas epidêmicas e indicadores epidemiológicos, nem ao processo dinâmico de disseminação e contágio, nem à "infodemia" de fake-news, mitos e mentiras, nem ao pânico que tudo isso provoca, nem às crises econômicas e políticas dela decorrentes ou a ela associadas. Trata-se de uma totalidade singular, específica e peculiar que no limite implica todos esses fenômenos e processos, em sua plena complexidade, articulados a outros elementos de compreensão e análise, unificados por meio de uma integralização heurística, que não se reduzem a medições, mediações, descrições, efeitos, correlações e narrativas (Fecher, 2020).

No início da pandemia Covid-19, pensadores e teóricos sociais de vários países do Hemisfério Norte se apressaram em demarcar territórios filosóficos e conceituais (Borri, 2020). Não obstante, por mais politicamente progressistas que algumas dessas análises pareçam, devemos assumir uma postura descolonizadora em relação a tais discursos (Santos, 2020). Para entender a pandemia e seus impactos, reais e imaginários, numa perspectiva realista e localizada em nosso próprio contexto, precisamos, mais que nunca, buscar referências conceituais, metodológicas e praxiológicas nas matrizes de pensamento do Sul global (Santos, 2004).

Nesse registro de insubmissão intelectual, há décadas uma epidemiologia crítica latino-americana tem-se dedicado a construir redes institucionais, referências científicas e caminhos acadêmicos independentes das formações hegemônicas das ciências modernas, bem estabelecidas no Norte global (Breilh, 2003). No Brasil, condicionada pelas contradições de uma formação social pe- 
riférica, desigual e diversa, a epidemiologia social vem sendo construída como subcampo de pesquisa e de prática, num registro de autonomia epistemológica, valorizando aspectos teóricos e políticos da Saúde Coletiva (Arreazza, 2012; Morabia, 2013).

O pressuposto central deste texto é que não há fundamentação lógica nem epistemológica no emprego dos conceitos de "modelo" e "modelagem" para designar exclusivamente dispositivos analíticos numéricos (Badiou, 2007) e que, pelo contrário, tal movimento revela-se inútil ou insuficiente para apreensão (e compreensão) da complexidade de eventos críticos como uma pandemia. Para responder à questão aqui posta, proponho tomar a pandemia Covid-19 como objeto complexo, com base numa proposta de modelagem heurística orientada por planos de ocorrência e interfaces hierárquicas. Para isso, em primeiro lugar, apresentarei uma súmula do pensamento de Juan Samaja [1941-2007], teórico argentino representativo de uma epistemologia da complexidade em nosso continente. Em segundo lugar, farei uma síntese da ideia de holopatogênese, elaboração conceitual diretamente derivada da teoria samajiana dos planos de emergência e interfaces hierárquicas, complementada com a contribuição de pensadores latino-americanos como Milton Santos, Gilles Bibeau e Néstor Garcia-Canclini. Terceiro, pretendo aplicar essa proposta de modelagem complexa para compreender a emergência da Covid-19 como tema-problema das ciências da saúde, visando formular uma perspectiva integradora capaz de contribuir para o enfrentamento e a eventual superação da pandemia. Finalmente, pretendo recorrer à categoria de sobredeterminação, para enriquecer esse esforço de compreensão teórica com um esquema organizador do conhecimento transdisciplinar sobre a pandemia Covid-19.

\section{Interfaces hierárquicas e multiplanidade}

Para uma modelagem da pandemia nos termos propostos, tomemos como plataforma epistemológica a obra de Samaja (1987, 1993, 1997, 2000, 2003, 2004). De início, Samaja (1997, p.213) destaca a natureza complexa, plural e, fundamentalmente, articuladora de múltiplas determinações do objeto das Ciências da Saúde, pois "implica un gran número de interfaces jerárquicas y enorme cantidad de información". Nessa abordagem, os fenômenos singulares da saúde-enfermidade-cuidado têm duas propriedades básicas: (a) ocorrem em distintos e simultâneos planos de emergência; (b) integralizam-se nas interfaces hierárquicas dos sistemas dinâmicos biológicos, ecossociais e socioculturais que conformam o mundo humano. Assim, o campo intertransdisciplinar das ciências da saúde estrutura-se sobre produções cognitivas dos distintos objetos subordinados, revelando diferentes planos de emergência e interfaces hierárquicas.

Para Samaja (1997, p.272), é preciso "[...] dialetizar la salud/enfermedad $y$ las prácticas que la constituyen, dejando lugar al reconocimiento de varios planos de emergência, en un sistema complejo de procesos adaptativos". Primeiro, num sentido recursivo, na medida em que existe-consiste em processos iterativos, re- 
produtores e transformadores das interfaces hierárquicas; segundo, num sentido conflitivo, posto que cada ordem hierárquica conserva um alto nível de autonomia em relação aos outros planos de ocorrência de eventos da saúde.

Numa apreciação inicial, Samaja $(1987$, p.80) considera pertinente distinguir entre indivíduo biológico e sujeito humano. Assim, visando à formulação de modelos explicativos da saúde desses entes históricos, as interfaces principais seriam: molécula-célula; célula-organismo; organismo-sociedade. Com a intenção de desdobrar a interface organismo-sociedade, indica distintas esferas da sociabilidade humana nas quais se realiza a reprodução social mediante processos de construção da saúde: "biocomunal, sociocultural, econômico-societal, ecológico-política" (Samaja, 2000, p.95).

A modo de síntese, a partir dessas proposições de Samaja, propus identificar três planos de emergência e suas respectivas interfaces hierárquicas (Almeida-Filho, 2011, p.135):

- subindividual (sistêmico/tissular/celular/molecular)

- individual (clínico/pessoal)

- coletivo (epidemiológico/populacional/social).

No plano subindividual, busca-se compreender processos biomoleculares em sistemas normais e suas anomalias e desvios como alterações, lesões ou desvios de função nos níveis microestruturais (moleculares, celulares), microssistêmicos (metabólicos, tissulares) ou macrossistêmicos (órgãos, sistemas corporais), a partir de cadeias de eventos com alto grau de estruturação. Já no plano de emergência individual, definidor do objeto da clínica médica (Almeida-Filho, 2000, p.91), objetos da saúde-doença-cuidado podem ser representados a partir de metáforas causais de variado grau de formalização, tomando o corpo humano como aquilo que Samaja (1993) denomina "nível de ancoragem". Nesse esquema, é possível situar no nível populacional a perspectiva epidemiológica convencional, fundada sobre uma lógica indutiva de base probabilística, no que se refere a doenças crônicas não transmissíveis, ou em modelos de cadeias de transmissão, em se tratando de doenças infectocontagiosas, como as síndromes respiratórias agudas.

Tomando o campo biológico como referência inicial, Samaja (2004) visualiza uma ordem hierárquica:

\section{[molécula-célula-tecido-órgão-sistema-organismo-grupo-população- -ambiente]}

No caso particular desses intrigantes organismos biológicos designados "seres humanos" inclui-se necessariamente a ordem simbólica. Assim, a série [organismo-grupo-população-ambiente] será lida como [sujeito-família-sociedade-cultura]. Em sintonia com Samaja, chamemos à primeira de ordem hierárquica biodemográfica e à segunda de ordem hierárquica sociocultural.

$\mathrm{Na}$ ordem hierárquica biodemográfica, conforme analisei em outro texto (Almeida Filho; Andrade, 2006, p.108), cada um dos campos disciplinares da 
biologia humana aplicada parece reivindicar a hegemonia do nível de ancoragem definidor do objeto saúde-doença. Uma versão simplista da biologia molecular toma o genoma (e seu derivado, o proteoma) como único tema válido e viável de pesquisa, argumentando que todos os outros níveis de processos patológicos derivam desses sistemas básicos de reprodução molecular e bioquímica. Assinalei que abordagens ingênuas e acríticas da histopatologia e da imunologia reivindicam a centralidade do nível tissular ou intrasistêmico na causalidade da patologia, assumindo que lesões e alterações constituem-se em causas materiais de sinais e sintomas que definem objetivamente o que é doença. Para dominar e controlar seu objeto de intervenção, o saber clínico toma a fisiopatologia como fonte de modelos explicativos de causalidade das doenças baseados em mecanismos regulatórios ou em desequilíbrios do sistema imune. Assim, cada campo disciplinar apresenta-se como único cientificamente válido e tecnologicamente aplicável para superar problemas de saúde, reduzindo os outros níveis de ocorrência e campos de saber a seus respectivos objeto e método.

Essa abordagem mal esconde uma pretensão de hegemonia científica, no sentido de que cada campo só aceita a contribuição de outro campo disciplinar sobre seu nível de ancoragem se esse se apresentar de modo subordinado, ou seja, colonizado. Uma aproximação a esse problema com mais consciência epistemológica pelo menos defenderia que o objeto "doença" é plural ou multifacetado, sendo simultaneamente defeito, lesão, alteração, patologia, doença, risco, dano, enfermidade (Almeida-Filho, 2011, p.143). A essa propriedade de ocorrência simultânea em múltiplos planos de emergência, descrita por Samaja, proponho designar como "multiplanidade".

$\mathrm{Na}$ ordem sociocultural, os fenômenos da vida, saúde, doença, sofrimento e morte se manifestam no espaço da linguagem, nas representações imaginárias, nos sistemas de crenças, nos modos de vida, enfim em todas as dimensões simbólicas e ideológicas de construção do objeto saúde-doença-cuidado. Para o estudo desses espaços e dimensões, sistemas de signos, significados e práticas de saúde abrem a possibilidade de incorporar tanto as respostas societais à enfermidade quanto a experiência privada e pessoal da doença (Bibeau, 2001). Isso implica incorporar no objeto-modelo saúde-doença e seus correlatos, por um lado, formas de reação das subjetividades humanas frente ao sofrimento e, por outro lado, modos de estruturação da representação social da saúde. De acordo com Bibeau (2001, p.792):

[...] representações, valores e conceitos relativos à saúde e à doença são inevitavelmente criados em um contexto de múltiplas formas de conhecimento, que se baseiam tanto nas epistemologias locais quanto estão ligadas ao contexto acadêmico mundial. Essas formas de conhecimento afetam as maneiras pelas quais os mundos da vida das pessoas são construídos e projetam a arquitetura daquela área nebulosa coberta pelo que cientistas sociais, com alguma hesitação, nomeiam de complexo saúde-doença-moléstia-enfermidade. ${ }^{1}$ 
Apesar de esforços localizados, o objeto de conhecimento dos vários campos disciplinares que constituem o chamado campo da Saúde tem sido em geral definido de modo inadequado (Almeida-Filho, 2013). Nesse engano, valoriza-se apenas a ordem hierárquica biodemográfica, negando-se qualquer função heurística à ordem hierárquica sociocultural. Reduzir a complexidade do objeto saúde-doença a conceitos unidimensionais como risco, doença e dispositivos ontológicos desse tipo, apesar das inegáveis aplicações para a construção de soluções tecnológicas eficazes e intervenções práticas efetivas, implica um ponto-cego, ou lacuna teórica, em relação ao núcleo epistemológico que precisamente define essa ordem de fenômenos como objeto de conhecimento (Almeida-Filho, 2000a).

\section{Totalidade e sobredeterminação}

Samaja (2003) propõe uma "epidemiologia miltoniana", capaz de lidar com complexos fenomênicos considerados como totalidades compostas por partes sobre as quais as ciências devem buscar um efeito sintetizador para preservá-los como integralidade heurística. Para Milton Santos (1996), construtos dessa natureza conformam uma nova família de objetos científicos, definidos não por estrutura, componentes, princípios funcionais e dimensões, mas sim por sua totalidade e transversalidade, em todos os planos de emergência. De fato, processos saúde-doença e fenômenos correlatos implicam uma classe peculiar de objetos-modelo sintéticos, ao mesmo tempo ontológicos e heurísticos, polissêmicos, polimórficos, plurais, enunciados para (e por) referência às dimensões fenomenológicas dos eventos em saúde. Como tal, não são vulneráveis à produção de conhecimento por meio da fragmentação (sendo, portanto, objetos adversos a processos analíticos), mas por meio de síntese e modelagem complexa (Hammond, 2003).

Objetos desse tipo tendem a maior grau de abstração para se tornarem objetos meta-sintéticos totalizados, o que ocorre após serem interpretados tomando como referência distintas camadas de realidade concretadas (ou imaginadas) pelas diversas ciências da saúde. Samaja (2003, p.115-6) destaca o termo "sobredeterminação" como conceito-chave para compreensão dessa questão:

Estas totalidades procesuales, a su turno, pueden pasar a formar parte de totalidades mayores, lo que supone que sus determinaciones propias son susceptibles de dirección o sobredeterminación, y lo que implica la supresión de su autonomia con conservación de su propio fundamento entitativo. (grifo meu)

Conforme Castiel (1988), a categoria "sobredeterminação" havia sido originalmente concebida por Sigmund Freud, ao formular uma teoria do que chamou aparelho psíquico ou aparelho de memória. Em outra oportunidade (Almeida-Filho; Coutinho, 2007), observamos que o conceito freudiano de sobredeterminação refere-se à série articulada de causas desencadeantes dos sintomas das neuroses e das diversas formações do inconsciente, compreendendo estímulos químicos, elétricos, energias e massas em movimento provenientes de 
fontes endógenas e exógenas. Posteriormente, o conceito de sobredeterminação seria por Freud vinculado à ideia de contingência, ao afirmar que se trata de um tipo de determinação definida por causas fracas, incluída juntamente com a condensação e o deslocamento como um dos mecanismos centrais da constituição dos sonhos e da gênese dos sintomas psíquicos (Almeida-Filho; Coutinho, 2007).

Essa ideia foi recuperada por eminentes formuladores de teorias críticas do conhecimento e da sociedade, como Bachelard, Bourdieu e sobretudo Althusser. Louis Althusser (2015), num capítulo intitulado "Contradição e sobredeterminação", apresenta a tese de que o conceito marxiano de contradição supõe uma sobredeterminação das diversas instâncias que compõem a estrutura social. Dessa maneira, encontra na sobredeterminação a possibilidade de uma lógica complexa, contrapondo-a à lógica linear, na qual o processo coincide com a produção e as instâncias de determinação são, a um só tempo, determinantes e determinadas, em constante retroalimentação. Dessa forma, nenhuma das instâncias ou conjunto de fatores pode ser reduzido a uma causa ou deduzida a partir dela.

Nessa mesma linha, Garcia-Canclini (2004, p.123) propõe uma "tradução cultural" da interpretação de Bourdieu sobre as múltiplas determinações que, para problemas concretos das ciências sociais, revelam sobredeterminação e não indeterminação. Em complemento, afirma que a "causalidade estrutural de uma rede de fatores" seria irredutível à eficiência aditiva de cada um desses fatores. Em suma, importa ressaltar neste aporte uma compreensão do caráter não binário e antiessencialista do conceito freudiano da sobredeterminação.

A conclusão, ainda provisória nessa altura, é que a categoria "sobredeterminação" mostra-se crucial para abordar a gênese estrutural de totalidades multifacetadas como um furacão ou uma pandemia. Conforme postulado por Samaja (2004), para aplicá-la com algum grau de eficiência na modelagem heurística de objetos complexos e singulares da ordem dos eventos críticos da saúde, precisamos identificar subespaços ou planos de ocorrência, introduzir formas de determinação, descrever objetos intermediários e delimitar interfaces estruturantes dos processos de totalização desses objetos. Em novas palavras, trata-se de formular a holopatogênese dos objetos complexos da saúde.

\section{Teoria da holopatogênese}

Em diálogo estreito com a obra de Samaja, vimos elaborando um esquema conceitual para uma teoria geral da saúde (Almeida-Filho, 2000, 2000a, 2001, 2006, 2011, 2013, 2014; Almeida-Filho; Coutinho, 2007), articulado a uma teoria restrita da doença denominada "holopatogênese", ${ }^{2}$ a qual apresento de modo resumido nesta seção. A categoria objeto-modelo, o esquema de multiplanos de ocorrência, a noção de modos de saúde e os conceitos de "Integrais de Saúde-Enfermidade" (ISE) e "complexo Moléstia-Enfermidade-Doença" (cMED) são elementos estruturantes dessa proposta teórica. 
Como referência axiomática para a modelagem de processos e fenômenos relacionados à saúde, consideremos os seguintes planos e componentes do objeto ISE, fazendo convergir as ordens biodemográfica e sociocultural:

- $[\mathrm{MSt}]$ microestrutural - molecular ou celular,

- $[\mathrm{MSy}]$ microssistêmica - metabolismo ou tecido,

- [SbI] subindividual (órgão ou sistema de corpo) - processos fisiopatológicos,

- [Cas] clínica individual - "casos",

- $[\mathrm{PaR}]$ epidemiológica - população sob risco,

- $[\mathrm{EcS}]$ ambiental - ecossistemas,

- [SiC] simbólica - semiológica e cultural

Consideremos ainda os seguintes pressupostos:

1. ISE refere-se ao conjunto real de fenômenos referidos à saúde que afetam corpos humanos, seres humanos e populações humanas; portanto, pode ser expresso como cMED que opera em distintos planos de ocorrência, onde eventos e processos ocorrem simultaneamente.

2. cMED não existe em si; portanto, fenômenos e processos de saúde emergem (ou passam a existir) se, e somente se, forem determinados por processos de holopathogênese (HPG).

3. HPG é um sistema gerador de estados, regido por processos de emergência e sobredeterminação; portanto, pode ser representado por uma rede de redes (net-network) em distintas interfaces hierárquicas e subespaços de ocorrência.

Cada nível de HPG implica componentes de uma determinada classe de determinação, com seus correspondentes ISE-estados. Nesse modelo, processos patológicos, estados de saúde e correlatos são sobredeterminados pelo complexo de processos e forças emergentes (causas, fatores, determinantes, vetores) ou pela ação articulada de diferentes conjuntos determinantes, e pelos valores assumidos por essas transformações em relação à resistência (capacidades, resiliência, força, entre outros) da dimensão afetada (célula, órgão, corpo, grupo, meio, entre outras). Na dimensão microestrutural MSt, HPG se expressa concretamente como processos moleculares ou celulares; na dimensão microssistêmica MSy, afeta metabolismo ou tecido; nos níveis subindividuais SbI, como processos fisiopatológicos em órgãos ou sistemas corporais; na dimensão clínica como casos individuais (Cas); na dimensão epidemiológica, como população sob risco $(\mathrm{PaR})$; ambientais nas interfaces dos ecossistemas EcS; no plano simbólico, em fundamentos semióticos ou socioculturais (SoC).

Conforme propus em outro texto (Almeida-Filho, 2014), processos HPG resultam da tensão entre agressores e defesas corporais componentes de redes de determinação, que genericamente podem ser distribuídas em dois subgrupos, patógenos e resistores. O primeiro grupo é formado por determinantes que pro- 
movem a ocorrência de componentes patológicos, os quais chamamos de holopatógenos. O segundo grupo inclui determinantes que aumentam a resistência à propagação da doença ou evitam a emergência de estados de não saúde no sistema, que designamos como resistores. Há permanente tensão dinâmica entre holopatógenos e resistores, ou $\mathrm{H}^{*} \mathrm{R}$, como mecanismos antagônicos, resultando em estados relacionados à doença como antirresistores AR: vulnerabilidade, suscetibilidade, fragilidades, debilidade, fraqueza, alteração, junto a outros elementos da HPG, conforme apresentado no Quadro 1.

Quadro l - Níveis e conceitos correlatos da holopatogênese

\begin{tabular}{l|c|c|c}
\hline $\begin{array}{l}\text { INTERFACES } \\
\text { HIERÁRQUICAS }\end{array}$ & HALOPATÓGENOS & ANTI-RESISTORES & DESFECHOS \\
\hline MSt & $\boldsymbol{\delta}$ Defeito & Debilidade: $\mathbf{d}$ & Alteração: Alt \\
\hline MSy & $\boldsymbol{\alpha}$ Agressão & Alteração: $\mathbf{s}$ & Lesão: Les \\
\hline Sbl & $\boldsymbol{\lambda}$ Lesão & Susceptibilidade: $\mathbf{S S}$ & Patologia: Pat \\
\hline Cas & $\mathbf{k}$ Causa & Susceptibilidade: $\mathbf{S V}$ & Doença Dis \\
\hline PaR & $\boldsymbol{\Phi}$ Fator & Vulnerabilidade: $\mathbf{V P}$ & Risco: $\mathbf{R S K}$ \\
\hline EcS & $\chi$ Condição & Vulnerabilidade: Ve & Dano: Haz \\
\hline SoC & $\boldsymbol{\sigma}$ Sentido & Fragilidade: $\mathbf{f}$ & Enfermidade: Sik \\
\hline
\end{tabular}

Fonte: Adaptado de Almeida Filho (2014).

O termo "alteração" refere-se a modificações na microestrutura de unidades orgânicas que, nos níveis molecular e celular, levam uma anomalia a se tornar anormal, ou um defeito. Os termos "debilidade" e "suscetibilidade" referem-se a processos similares que correspondem às dimensões ISE microssistêmica e subindividual, respectivamente. Conceitos de suscetibilidade têm sido amplamente utilizados nos campos da medicina, epidemiologia, biologia, ecologia, engenharia e toxicologia, implicando diferentes ênfases que se relacionam com as perspectivas e métodos subjacentes de cada campo (Najman, 1980; Parkin; Balbus, 2000).

Prioridade especial deve ser concedida ao conceito de vulnerabilidade e seus correlatos, particularmente eficaz na dimensão social e na esfera subjetiva (Ayres et al., 2003). Isso vale também para conceitos de fragilidade, que já possuem status teórico bastante reconhecido na pesquisa de ecossistemas e nos modelos de determinação social da doença (Niero, 2012; Grabovschi; Loignon; Fortin, 2013).

Dadas essas definições, a noção de desfechos pode ser introduzida no esquema da HPG. Consideremos a seguinte notação para os resultados da HPG, nos distintos planos de ocorrência: defeito - Def; lesão - Les; patologia - Pat; doença (ou desordem) - Dis; risco - Rsk; dano - Haz; doença - Sik. Esse esque- 
ma pode ser útil como pano de fundo para um modelo generalizado do "complexo Moléstia-Enfermidade-Doença" (cMED) como uma rede de processos de determinação de fenômenos relativos à atenção à saúde-doença nas populações humanas, em subespaços ou níveis distintos de ocorrência, mediados por relações antagônicas de patogênese e salutogênese.

As diferentes dimensões da HPG compartilham determinantes e componentes dos outros subespaços. Essa situação é implicitamente reconhecida no Quadro, onde conceitos como alteração e lesão aparecem em diferentes espaços de HPG, seja como um estado ISE característico do cMED ou como determinante de estados de saúde da doença no nível subsequente. Nesse quadro, o conceito da HPG pode ser interpretado como um tipo especial de objeto-modelo heurístico, capaz de operar em diferentes níveis hierárquicos de complexidade, simultaneamente dependentes de substratos ontológicos e simbólicos (Samaja, 2004). Tal modelo pode ser aplicado a diferentes classes de ISE-estados, que possuem uma ou mais das facetas referidas.

Proposto para lidar com problemas teóricos e metodológicos normalmente evitados por paradigmas tradicionais da patogênese, tal arcabouço conceitual requer integração de diversas abordagens disciplinares num esforço de pesquisa articulado e interativo. Consideremos o sinal \# como notação para uma dada interface hierárquica. Os subespaços intertransdisciplinares, e as respectivas regras de determinação, podem ser representados como contiguidades ou correspondências entre os níveis de objeto, organizados como interfaces: biomolecular\#imunológico (molécula para célula), fisiopatológico\#clínico (órgão/sistema para corpo), epidemiológico\#ecossocial (população para espécie). Certamente, a ordem simbólica permeia (e sobredetermina) todos os conjuntos, subespaços e planos de emergência dos complexos fenomênicos da saúde-enfermidade-cuidado, tal como ocorre nas pandemias e outros eventos epidemiológicos críticos.

A representação de facetas distintas de um objeto ontológico total como o ISE exige modelos heurísticos capazes de operar na multiplanidade, como o são os modelos HPG, simultaneamente ontológicos e heurísticos, plurais, polissêmicos, multifacetados (Bunge, 1980). Como tal, recortam padrões e domínios distintos referentes a diferentes componentes e níveis de complexidade. Modelos biológicos de determinação operam componentes e processos estruturais ou sistêmicos, em diferentes níveis de ocorrência (molécula, célula, tecido, órgãos e sistemas). Modelos clínicos baseiam-se em quadros explicativos mecanicistas ou causais referidos ao nível individual (casos de doença). Modelos epidemiológicos pseudoprobabilísticos baseados no conceito de risco e ecossociais subsidiados pela noção de dano (bazard) têm como referentes os níveis populacional e ambiental (Krieger, 2001). Modelos linguísticos e simbólicos de cuidados com a saúde estão ancorados no domínio da linguagem e da cultura (Bibeau, 2001). 
A categoria "sobredeterminação" será útil para ajudar a compreender a cadeia de componentes, fatores e vetores que conformam o cMED como objeto científico do conhecimento. Nesse referencial teórico, redes de processos múltiplos e simultâneos de sobredeterminação geram trajetórias de patogênese dinâmicas e complexas.

\section{Holopatogênese da Covid-19}

A pandemia Covid-19 constitui-se, sem dúvida, num caso de evento crítico de saúde, revelando múltiplas interfaces entre planos de ocorrência, com vários objetos intermediários estruturantes do que será um complexo fenomênico totalizado. Segundo a epistemologia samajiana da complexidade (Samaja, 2003; 2004), objetos-modelo em saúde podem ser prototípicos para uma desejada integração entre ciências biomoleculares (geradoras de conhecimentos da base físico-química-biológica), ciências clínicas (responsáveis pela modelagem não numérica dos desfechos em saúde), ciências da saúde pública (produtoras de conhecimento de base demográfica ou populacional, tipicamente sob a forma de modelagem numérica de riscos e fatores), ciências sociais (por exemplo, com os processos macrossociais representados como composição de elementos na base do modelo), lógica e semântica (para validar vínculos simbólicos entre padrões, vetores e determinantes expressos em submodelos e modelos sintéticos).

O complexo "Moléstia-Enfermidade-Doença" dessa pandemia, ou cMED!Covid-19, pode ser parcialmente definido como um conjunto articulado de facetas de patogênese nos planos subindividuais, de causalidade no plano clínico individual, de determinação nos planos coletivos, de produção semântica nos planos simbólicos. Cada combinação de plano e seu respectivo desfecho compõem um modelo ontológico parcial da pandemia, ou um submodelo de patogênese dos fenômenos integrais de saúde-enfermidade-cuidado implicados. Modelagens reduzidas deste primeiro objeto ontológico da Covid-19 podem agregar facetas e dimensões diversas como se todas elas pertencessem à mesma classe e ordem, como na simples soma de componentes da patogênese numa modelagem aditiva de $[$ Def + Les + Pat + Dis + Rsk + Haz + Sik $]$.

Conforme a notação apresentada no Quadro 1, dentro dos parênteses, são apontados diferentes fatores de subconjuntos $\mathrm{H}^{*} \mathrm{R}$ que alteram o complexo sistema de estados patológicos cMED, de $\delta$ a $\sigma$. Nesta modelagem, $(\delta \ldots \sigma \rightarrow)$ é a notação geral para vínculos ou nexos entre determinantes e desfechos em todos os níveis, componentes e padrões de holopatogênese, discriminados como segue:

1. modelos genéticos microestruturais da infeção por Sars-CoV-2 produzem anomalias moleculares ou celulares, levando a danos ou defeitos celulares Def, ou ( $\rightarrow$ Def!Sars-CoV-2);

2. modelos microssistêmicos de infeção por Sars-CoV-2 produzem ajustes imunológicos, desvios metabólicos ou defeitos teciduais, resultando lesões Les, ou ( $\rightarrow$ Les!Sars-CoV-2); 
3. modelos fisiopatológicos representam processos subindividuais de reação à infeção por Sars-CoV-2, manifestos na forma de lesões que criam condições para sua expressão como patologias, ou ( $\rightarrow$ Pat!Covid-19);

4. modelos clínicos da Covid-19 podem ser construídos com base em danos de órgãos ou falhas dos sistemas corporais, conhecidos como causas que produzem casos de coronavirose, ou Cas!Covid-19, ou $(\rightarrow$ Dis!Covid-19);

5. modelagem numérica com indicadores epidemiológicos incorpora fatores de exposição e grupos de risco, determinando riscos, ou risco de adoecimento pela Covid-19, ou ( $\rightarrow$ Rsk!Covid-19), induzindo ações preventivas;

6. modelos ecossistêmicos de saúde incluem condições de possibilidades de contágio ou cadeias de transmissão, levando a danos Haz!Covid-19, ou $(\rightarrow \mathrm{Haz})$, fomentando componentes precaucionários;

7. modelos narrativos semióticos operam mediante interação de sistemas de signos/significados/práticas, gerando percepção social de enfermidade Sik!Covid-19 mediada por dispositivos simbólicos SoC, ou $(\rightarrow$ Sik).

Modelos desse tipo são uma representação parcial e incompleta da complexidade do sistema cMED dos IES-estados na medida em que não permitem a articulação entre os submodelos de cada dimensão ou modelagem dos subespaços da HPG! Covid-19. Se os subespaços da HPG!Covid-19 fossem estritamente ortogonais, a evolução dinâmica de cada submodelo dimensional seria independente dos outros ocorridos nas respectivas interfaces hierárquicas.

A partir desse patamar inicial, podemos derivar um modelo geral de holopatogênese com a condensação de todos os subespaços de patogênese considerados, gerando assim um modelo geral do cMED!Covid-19. Na formalização do objeto HPG!Covid-19, o símbolo $\Phi$ indica que cada um dos componentes deve ser definido em subespaços distintos, pertencentes a planos ortogonais, num espaço lógico geral formado pelo produto cartesiano dos subespaços nas interfaces hierárquicas.

HPG!COVID-19: $[f \operatorname{MSt}(\alpha \rightarrow$ Def $)] \Phi[f \operatorname{Msy}(\delta \rightarrow$ Les $)][f \operatorname{SbI}(\lambda \rightarrow$ Pat $)]$ $\Phi[f \mathrm{Cas}(\mathrm{k} \rightarrow \mathrm{Dis})] \Phi[\operatorname{PaR}(\Phi \rightarrow \mathrm{Rsk})] \Phi[f \mathrm{EcS}(\chi \rightarrow \mathrm{Haz})][f \mathrm{SoC}(\sigma \rightarrow \operatorname{Sik})]$

Esse sistema de subespaços ou planos de ocorrência pode ser graficamente (e metaforicamente) representado como um mosaico de redes, articuladas numa rede de redes. Tendo como referente múltiplas ocorrências simultâneas, é possível construir uma modelagem sintética, ao mesmo descritiva e gráfica de uma determinação ainda segmentada e linear do complexo fenomênico Sars-CoV-2 $\Phi$ Covid-19 $\Phi$ Epidemia $\Phi$ Pandemia $\Phi$ Infodemia, conforme a Figura 1.

$\mathrm{Na}$ perspectiva de modelagem de objetos ontológicos complexos como a pandemia Covid-19, subespaços distintos exigem submodelos heurísticos correspondentes, interconectados em torno de um núcleo comum de acoplamento 
estrutural do cMED. Esse esquema gráfico e os que seguem representam ilustração do argumento, sem qualquer pretensão de modelagem orientada por dados empíricos ou mesmo simulados.

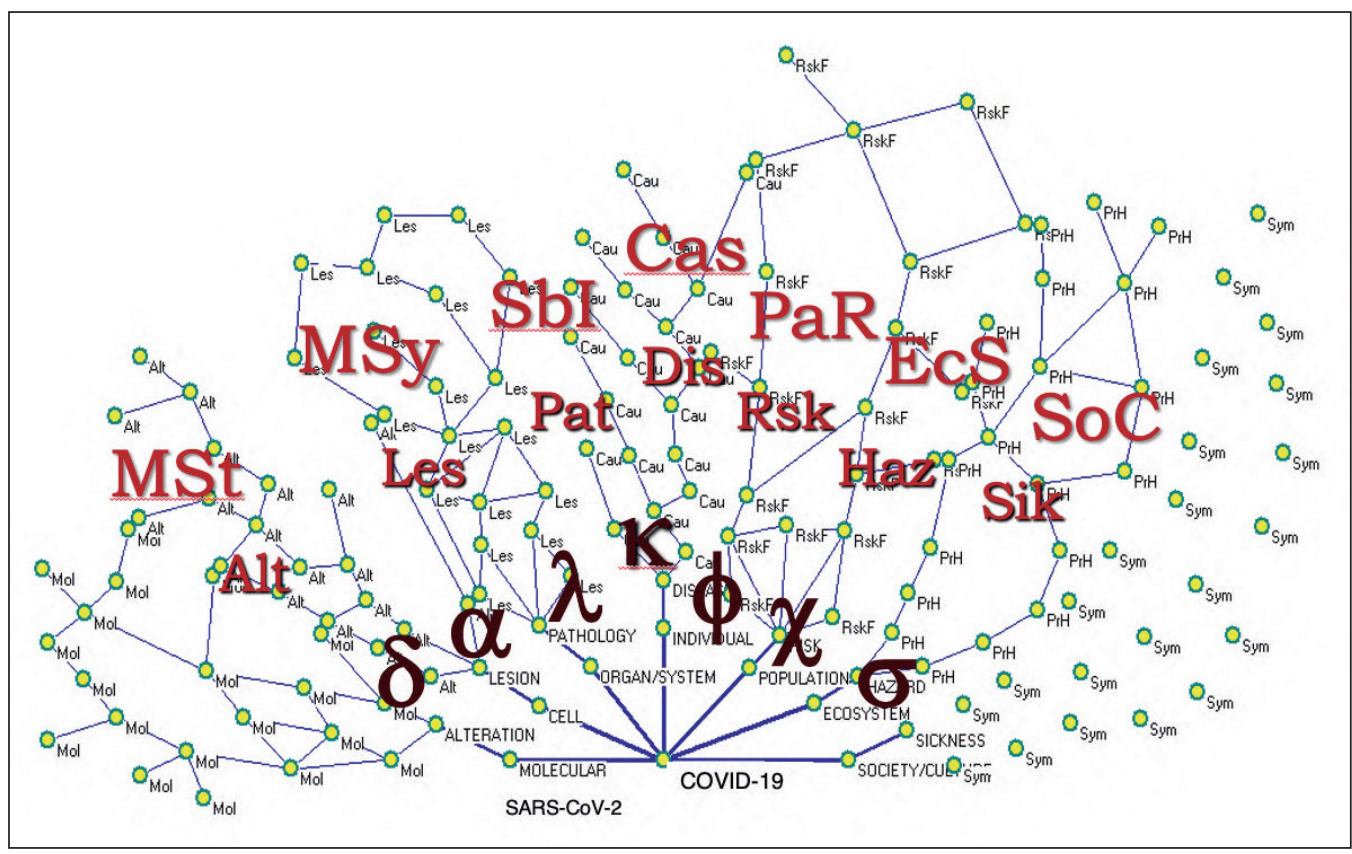

Fonte: Adaptado de Almeida Filho (2014).

Figura 1 - Subespaços HPG, rede de redes e desfechos da Covid-19.

De todo modo, uma compreensão rigorosa da holopatogênese admite a possibilidade de fusão, combinação, composição ou articulação de lógicas de determinação distintas, reforçando o argumento de que nem todos os modelos podem ser expressos na forma algébrica ou, para usar uma linguagem mais direta, reduzidos à mera quantificação. Portanto, uma modelagem mais precisa e eficiente do cMED!Covid-19 precisa ultrapassar a modelagem numérica, incorporando a projeção de redes de determinação sobre subespaços distribuídos em trajetórias convergentes num holoespaço, mediante processos que, como vimos em seções anteriores, têm sido definidos como sobredeterminação.

\section{Sobredeterminação da pandemia Covid-19}

Para uma modelagem eficiente de objetos complexos em saúde, permitindo operar metodologicamente estratégias de produção de conhecimento e controlar seus efeitos mediante intervenções para transformação de realidades, propusemos recorrer à categoria de "sobredeterminação" (Almeida-Filho; Coutinho, 2007). Atualizando essa contribuição, proponho utilizar três sentidos operacionais para o termo sobredeterminação: a) trajetórias de contingências; b) categoria mais geral numa taxonomia de processos determinantes; c) rede heurística em modelos de complexidade. 
No primeiro sentido, a sobredeterminação de fenômenos complexos se revela nas cadeias de eventos ou trajetórias de determinação desencadeadas ou provocadas por acidente ou contingência, abertas a outras modalidades de determinação (Bunge, 1980). Entre as propriedades, destacam-se (i) a multideterminação ou causalidade plural; (ii) a impossibilidade de efeito preditivo, pois suas determinações apresentam-se por retroação, o que significa dizer que são reconstituídas a posteriori; (iii) uso de lógicas não indutivas, podendo ser dedutiva ou demonstrável e abdutiva.

A segunda acepção de sobredeterminação se revela em sua natureza polissêmica, permitindo defini-la como expressão geral dos processos determinantes, organizados no seguinte referencial semântico: "causação" biológica de patologias, "determinação" social da situação e das condições de saúde, "produção" cultural das práticas de saúde, "construção" política das instituições e sistemas de saúde e "invenção" simbólica dos sentidos da saúde.

$\mathrm{Na}$ terceira referência, condensando as acepções anteriores, Redes de Sobredeterminação (RSD) permitem a modelagem complexa de eventos singulares e multiplamente determinados, desencadeados por contingências e emergências, referenciáveis por modelos causais, fatores de risco ou processos estocásticos. Para se expressar no registro da RSD, o modelo da HPG! Covid-19 deve ser reformulado a fim de incorporar as propriedades de singularidade e multiplanidade desse evento crítico e suas contingências, incorporando classes específicas de determinantes e soluções de acoplamento estrutural entre submodelos de patogênese, de causalidade, de determinação e, no conjunto complexo, de sobredeterminação.

Sem qualquer pretensão de apresentar uma formalização algébrica restrita, a articulação de elementos sobredeterminados pode ser representada pelo sinal $\Leftrightarrow$ tendo como referência cada um dos submodelos da HPG, da seguinte forma:

HPG!COVID-19: $\left[f_{1} \operatorname{MSt}(\alpha \rightarrow\right.$ Def $\left.)\right] \Leftrightarrow\left[f_{2} \operatorname{Msy}(\delta \rightarrow\right.$ Les $\left.)\right] \Leftrightarrow\left[f_{3} \operatorname{SbI}(\lambda \rightarrow\right.$ Pat $\left.)\right]$ $\Leftrightarrow\left[f_{4} \operatorname{Cas}(\mathrm{k} \rightarrow \mathrm{Dis})\right] \Leftrightarrow\left[f_{5} \mathrm{PaR}(\Phi \rightarrow \mathrm{Rsk})\right] \Leftrightarrow\left[f_{6} \mathrm{EcS}(\chi \rightarrow \mathrm{Haz})\right] \Leftrightarrow\left[f_{7} \mathrm{SoC}(\sigma \rightarrow \operatorname{Sik})\right]$

Reunindo conhecimento gerado em poucos meses de pesquisa sobre o patógeno Sars-CoV-2 nos planos moleculares subindividuais e sobre os efeitos clínicos do cMED!Covid-19 no plano individual, juntamente com a modelagem dos processos de determinação via cadeias de contágio, conhecidos há séculos e décadas, o modelo geral da HPG!Covid-19 pode ser visualizado como uma rede de redes para a sobredeterminação da pandemia Covid-19.

A Figura 2 é uma representação gráfica desse modelo sistêmico-dinâmico geral da HPG!Covid-19, rede de redes sobredeterminada, interconectada em todas as suas dimensões, holopatógenos e desfechos, articulada numa ordem complexa não hierárquica, porém respeitando as interfaces hierárquicas indicativas da diversidade dos nexos determinantes. O destaque visual e a posição central da enfermidade em foco, na rede de conexões de determinação, são artefatos gráficos, claramente arbitrários, podendo esta se colocar em qualquer ponto da rede. 


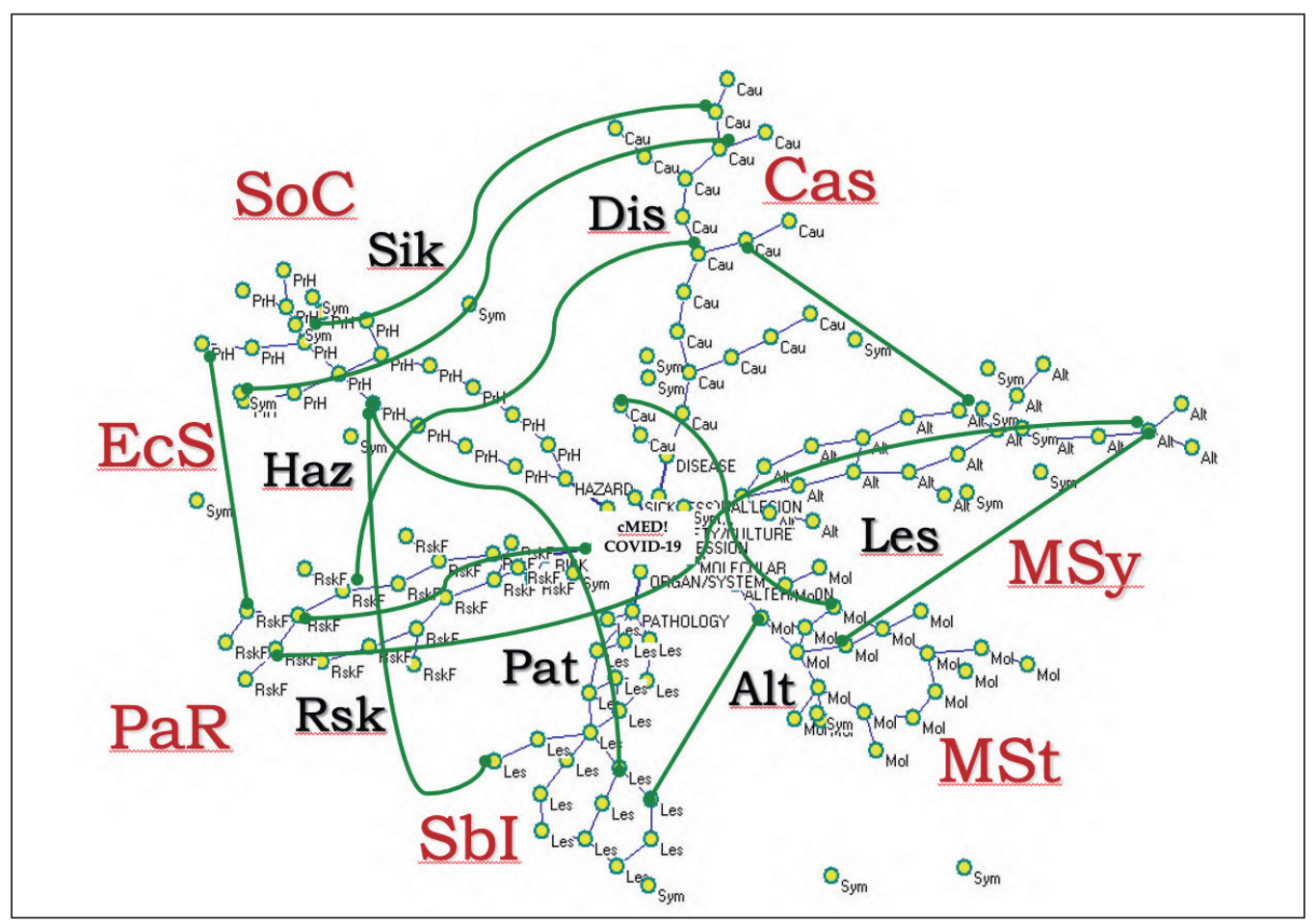

Fonte: Adaptado de Almeida Filho (2014).

Figura 2 - Rede de redes e acoplamento estrutural da HPG!Covid-19.

Muitos dos links internos aos submodelos nos subespaços pertencem ou estão conectados a outras cadeias de determinação da HPG!Covid-19, em diferentes níveis ou domínios do cMED!Covid-19. Tais relações são articuladas por processos simultâneos de "sobredeterminação" das diferentes classes do cMED.

Conforme assinalamos em outra oportunidade (Almeida-Filho; Andrade, 2006), por serem quase ortogonais, os subespaços HPG estão inter-relacionados mediante nexos de "acoplamento estrutural", de tal forma que a projeção do subespaço (i) no outro subespaço (j) afeta os efeitos individuais dos subconjuntos Hi e Ri sobre o estado patogênico do nível j. Essa influência será então exercida através de uma projeção do conjunto desses determinantes, que pode ser definida, em uma primeira abordagem, pelos valores assumidos pela variável estadual de nível i. De fato, o desafio de oferecer uma interprojeção adequada de efeitos em diferentes escalas na modelagem de fenômenos de doenças de saúde é uma demanda não trivial para as várias tentativas de abordagem de sistemas complexos.

As formas elementares dessas operações podem ser extraídas dos dados empíricos disponíveis em cada dimensão/nível/faceta da HPG! Covid-19, tanto quanto da precisão com que diferentes modelos poderão reproduzir e prever situações observadas em situações concretas de saúde. Nesse sentido, o mapeamento da HPG também permite a representação visual da influência das distintas categorias e territórios epistêmicos no modelo cMED!Covid-19, conforme ilustrado na Figura 3. 


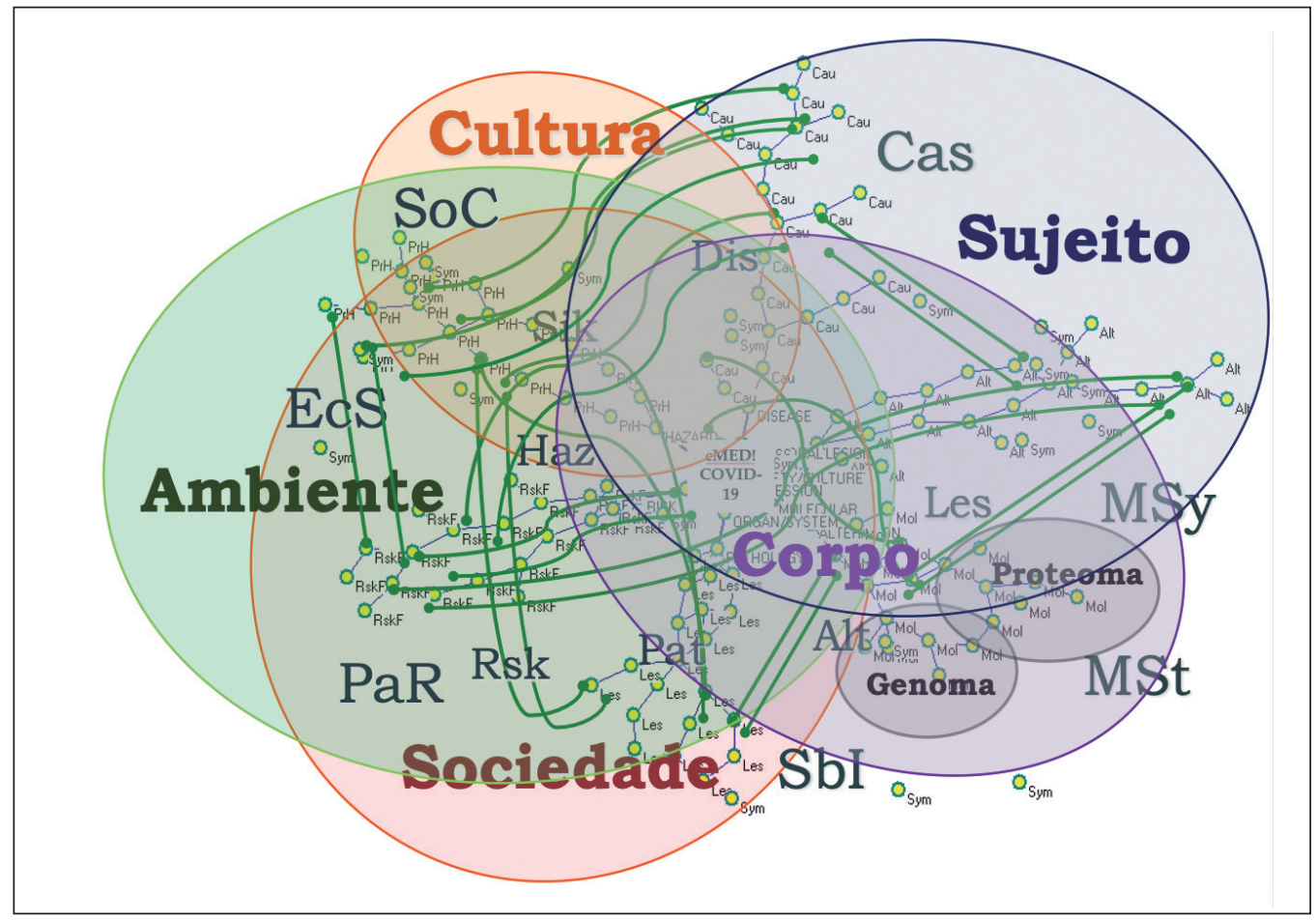

Fonte: Adaptado de Almeida Filho (2014).

Figura 3 - Rede de redes e acoplamento estrutural da HPG!Covid-19.

Na Figura 3 se demonstra graficamente, ainda que de modo esquemático, quão limitados em escopo e relevância, para a sobredeterminação dos desfechos que definem natureza e alcance da pandemia Covid-19, são os alvos de pesquisa no subespaço biomolecular em comparação com as dimensões clínicas, ambientais, socioculturais, econômicas e políticas. Notadamente no que se refere à pandemia Covid-19, note-se que os subespaços do corpo orgânico, do sujeito humano, da sociedade, do ambiente e da cultura, superpostos e articulados, em muito superam o potencial explicativo dos focos atualmente mais explorados nos contextos científicos contemporâneos: o complexo genoma-proteoma.

A infeção por Sars-CoV-2, evento contingente, produz uma determinada patologia (ou defeito, desordem, lesão, perturbação ou anormalidade) capaz de provocar danos ou falhas em um órgão-alvo, num sistema corporal. O patógeno Sars-CoV-2 pode ser classificado como causa de uma doença, clinicamente reconhecida e biologicamente identificada, designada como Covid-19. O aumento acelerado de infectados e casos clínicos, assintomáticos ou não, a circulação do vírus numa determinada população, ou numa sociedade, a transmissão em redes sociais, a concentração demográfica ou a agregação de sujeitos, podem representar fatores de risco ou ameaça ambiental para doenças transmissíveis, como é o caso da Covid-19. Fatores geopolíticos, relações econômicas ou vetores migratórios são capazes de transformar um surto epidêmico numa pandemia, ao tem- 
po em que ondas de informações falsas e decisões políticas equivocadas podem resultar em redução da capacidade de controle do contágio.

É importante notar que todos esses eventos ocorrem ao mesmo tempo, em distintos planos de emergência ou subespaços do espaço multiplano e transdimensional da HPG!Covid-19. Contemplando ocorrências simultâneas, podemos delinear uma modelagem heurística de uma série de formas distintas de determinação do complexo fenomênico Sars-CoV-2 $\Phi$ Covid-19 $\Phi$ Epidemia $\Phi$ Pandemia $\Phi$ Infodemia, conforme apresentado no Quadro 2.

Quadro 2 - Interfaces e elementos da modelagem heurística da holopatogênese

\begin{tabular}{l|c|c|c}
\hline $\begin{array}{l}\text { INTERFACES } \\
\text { HIERÁRQUICAS }\end{array}$ & Objetos & Determinação & Modos de intervenção \\
\hline Biomolecular // Clínica & SARS-CoV-2 & Infecção & Imunização \\
\hline Clínica // Epidemiológica & CoViD-19 & Causa & Tratamentos \\
\hline Epidemiológica // Ecossocial & Pandemia & Risco & Vigilância \\
\hline Ecossocial // Tecnológica & Epidemias & Dano & Controle \\
\hline Tecnológica // Econômica & Economia & Recessão & Proteção social \\
\hline Econômica // Política & Estado & Crise & Legislação \\
\hline Política // Simbólica & Infodemia & Disseminação & Mobilização \\
\hline
\end{tabular}

A correspondência entre interfaces hierárquicas, objetos de conhecimento específico (ou subobjetos em relação ao objeto-modelo complexo) e formas de sobredeterminação se estende aos modos de intervenção viáveis em cada plano de ocorrência dos processos biológicos, clínicos, epidemiológicos, ecológicos, tecnológicos, políticos e culturais que compõem o complexo fenomênico da pandemia Covid-19. Na interface Biomolecular\#Clínica, onde o patógeno Sars-CoV-2 atua causando patologia, contágio, doença e eventualmente falência de órgãos e sistemas, mediante processos de infecção, o modo de intervenção consiste na indução ou animação do sistema imunológico dos corpos individuais, no nível subindividual (ou no nível coletivo, com as vacinas, por exemplo). Na interface Clínica\#Epidemiológica, onde ocorre a causação da enfermidade Covid-19 e eventualmente a morte, modos de intervenção compreendem tratamentos visando à cura dos sujeitos ou redução de letalidade e sequelas da doença. Na interface Epidemiológica\#Ecossocial, onde cursa a pandemia da Covid-19, determinantes sociais transformam casos infectados e infectantes em grupos de risco e de vulnerabilidade, indicando amplas e efetivas medidas de vigilância epidemiológica para redução de incidência e controle da transmissibilidade. Na interface Ecossocial\#Tecnológica, a pandemia se transmuda em sistemas de epidemias alimentados por cadeias e ondas de contágio, estressando a capacidade da sociedade em produzir conhecimentos e novas tecnologias. $\mathrm{Na}$ 
interface Tecnológica\#Econômica, é indispensável reduzir desigualdades e iniquidades, eliminando discriminações inaceitáveis no acesso aos cuidados de saúde, bem como a adesão às medidas de isolamento físico/social. Ante a pandemia e a recessão econômica dela derivada, essa interface exige a adoção de medidas econômicas emergenciais que assegurem a proteção social. A interface Econômica\#Política pressupõe relações de credibilidade entre as autoridades sanitárias e políticas e a população, sendo imprescindível assegurar qualidade, transparência e acesso às informações em saúde para a construção de estratégias e a tomada de decisão no combate à pandemia. Finalmente, na interface Política\#Simbólica, intensa produção informacional e narrativa se dissemina e alimenta um imaginário social cheio de ansiedade e medo (Santos, 2020), no que se denominou de "infodemia", desencadeando estratégias de mobilização e engajamento de sujeitos, grupos e massas.

\section{Comentários finais}

Samaja (2004) comenta que, no contexto de uma ontologia formal da saúde na evolução humana, a teoria da holopatogênese pode ser considerada uma transdisciplina fundada numa teoria unificada da saúde-doença-cuidado. Nela encontra-se a possibilidade de incorporação analítica de propriedades de objetos específicos e referentes (por exemplo, a um tecido, a um sistema imunológico, a uma distribuição populacional, a um programa de saúde ou a um sistema de cuidados) para a compreensão sintética de um dispositivo heurístico total, melhor definido em termos de densidade, funcionalidade e diversidade. Abordagens dessa ordem seriam mais eficientes do que modelos explicativos obtidos por equações diferenciais parciais ou modelagens numéricas aplicadas a seções isoladas de uma cadeia fragmentada de causalidade (Kothari; Mehta, 1981).

A HPG de um dado cMED tipicamente tem como nível de ancoragem o plano clínico, porém a natureza de epidemia ou pandemia desse evento crítico permite estabelecer o nível de ancoragem da HPG!Covid-19 no plano ecossocial. Apesar do fetiche das curvas epidêmicas, sobretudo no contexto da infodemia que, de certo modo, tornaria o plano político-simbólico um importante atrator do nível de ancoragem, a Epidemiologia se apresenta como o principal campo produtor de significantes sobre a pandemia Covid-19. Essa constatação tem produzido, como efeito imediato, uma peculiar disputa com outros campos de conhecimento, em dois sentidos: por um lado, entre pesquisadores e produtores de discurso técnico do campo médico, muitos se declaram epidemiologistas, mesmo quando abertamente demonstram reduzida ou enviesada compreensão do raciocínio epidemiológico; por outro lado, pesquisadores de campos científicos e tecnológicos estruturados nas diversas lógicas de quantificação, como a física, a economia, a estatística, a computação e o que, agora na moda, chamam de "ciências de dados", agem como se nunca houvesse existido um campo disciplinar específico, constituído a partir do estudo das epidemias, que historicamente se desenvolveu tendo como eixo epistemo-metodológico 
abordagens numéricas da distribuição de doenças e fenômenos de saúde em populações, ambientes e sociedades.

Nesse contexto atual de intensa disputa retórica e renhida luta teórica, a singularidade e complexidade da atual pandemia da Covid-19 sem dúvida representa rica oportunidade para realizar de modo efetivo a construção intertransdisciplinar tão necessária para a geração de soluções integradoras, pertinentes e cuidadosas ante os problemas complexos que emergem nos diversos planos e dimensões dessa grave crise sanitária. Particularmente no Brasil, no que diz respeito aos processos concretos da realidade de saúde, esforços criativos voltados à produção de modelos sintéticos dessa ordem podem ser pertinentes para a construção de objetos complexos transdisciplinares. Espero que estas anotações samajianas de algum modo contribuam para melhor diálogo e articulação entre as diferentes disciplinas e campos das ciências no que pode ser um ganho secundário dessa grave crise sanitária, a ser considerado no desenvolvimento de novas e promissoras estratégias de pesquisa sobre a HPG!Covid-19.

\section{Notas}

1 No original: "representations, values, and concepts concerning health and disease are inevitably created within a context of multiple forms of knowledge which are as much grounded in local epistemologies as they are linked to the scholarly academic world. These forms of knowledge affect the ways by which the life-worlds of persons are built and design the architecture of that fuzzy area covered by whatsocial scientists name, with some hesitation, the health-disease-illness-sickness complex" (tradução do autor).

2 Etimologicamente, o conceito de "holopatogênese" se define como conjunto ou rede de processos de sobredeterminação (gênese) de doenças, doenças e condições relacionadas (pathos) tomados como um objeto integral, complexo, total (holos), compreendendo facetas distintas, manifestações e expressões de tal objeto de conhecimento que opera em distintos níveis hierárquicos de complexidade, dependentes de seu tema ontológico e simbólico (Almeida-Filho; Andrade, 2006).

\section{Referências}

ALMEIDA-FILHO, N. La ciencia tímida-Ensayos de deconstrucción de la epidemiologia. Buenos Aires: Lugar Editorial, 2000.

. O conceito de saúde: ponto-cego da epidemiologia? Revista Brasileira de Epidemiologia, v.3, n.1-3, p.4-20, 2000a.

. For a general theory of health: preliminary anthropological and epistemological notes. Cadernos de Saúde Pública, v.17, n.4, p.753-70, 2001.

. Complejidad y transdisciplinariedad en el campo de la Salud Colectiva: evaluación de conceptos y aplicaciones. Salud Colectiva, v.2, p.123-46, 2006.

. O que é Saúde? Rio de Janeiro: Editora Fiocruz, 2011.

. Towards a Unified Theory of Health-Disease: I. Health as a complex model-object. Rev. Saúde Pública, v.47, n.3, p.433-50, 2013. 
ALMEIDA-FILHO, N. Towards a unified theory of health-disease: II. Holopathogenesis. Rev. Saúde Pública, v.48, n.2, p.192-205, 2014.

ALMEIDA-FILHO, N.; ANDRADE, R. Holopatogénesis: Esbozo de una teoría general de salud-enfermedad como base para la promoción de la salud. In: CZERESNIA, D.; FREITAS, C. M. de. (Org.). Promoción de la salud. Conceptos, reflexiones, tendencias. Buenos Aires: Editorial Lugar, 2006. p.113-34.

ALMEIDA-FILHO, N.; COUTINHO, D. Causalidade, contingência, complexidade: o futuro do conceito de risco. Physis. Revista de Saúde Coletiva, v.17, p.95 -137, 2007.

ALTHUSSER, L. Contradição e sobredeterminação. In: Por Marx. Campinas: EdUnicamp, 2015[1967]. p.71-95.

ARREAZA, A. L. V. Epidemiologia crítica: por uma práxis teórica do saber agir. Ciênc. saúde coletiva [online], v.17, n.4, p.1001-13, 2012.

AYRES, J. R. M. et al. O conceito de vulnerabilidade e as práticas de saúde: novas perspectivas e desafios. In: CZERESNIA, D.; FREITAS, C. M. de. Promoção da saúde: conceitos, reflexões, tendências. Rio de Janeiro: Fiocruz, 2003. p.117-39.

BADIOU, A. Le concept de modèle. Paris: Fayard, 2007.

BIBEAU, G. In defense of a creolized grammar of the health-disease complex. Cad. Saude Pública [Internet], v.17, n.4, p.787-92, ago. 2001. Disponível em: <http://www.scielo. br/scielo.php?script=sci_arttext\&pid=S0102-311X2001000400013\&lng=en $>$. Acesso em: 31 maio 2020.

BORRI, N. (Org.) Sopa de Wuban, Pensamiento contemporáneo en tiempos de Pandemias. Giorgio Agamben, Slavoj Zizek, Jean Luc Nancy, Franco Berardi, Santiago Lopez Petit, Judith Butler, Alain Badiou, David Harvey, Byung-Chul Han, Raul Zibechi, Maria Galindo, Markus Gabriel, Gustavo Yanez Gonzalez, Patricia Manrique, Paul Preciado. Buenos Aires: ASPO (Aislamiento Social Preventivo y Obligatorio), 2020. 288p.

BREILH, J. Epidemiología critica: ciencia emancipadora e interculturalidad. Buenos Aires: Lugar Editorial, 2003.

BUNGE, M. Epistemología: ciencia de la ciencia. Barcelona: Ariel, 1980.

CASTIEL, L. D. Freud: um epidemiologista? Cad. Saúde Pública [online], v.4, n.3, p.316-25, 1988.

DAS, V. Critical Events. An Anthropological Perspective on Contemporary India. New Delhi: Oxford University Press, 1996.

DAUPHINÉ, A.; PROVITOLO, D. La complexité des risques et des catastrophes. In: DAUPHINÉ, A.; PROVITOLO, D. (Ed.) Risques et catastrophes. Observer, spatialiser, comprendre, gérer. Paris: Armand Colin, 2013. p.209-36.

ELLIOT, N. L. Mediating Nature. London: Routledge, 2006.

FECHER, B. Embracing complexity: Covid-19 is a case for academic collaboration and co-creation. Elephant in the lab, 2020. Disponível em: <https://doi.org/10.5281/ zenodo.3712898>.

FREUD, S. El mecanismo psíquico de los fenómenos histéricos (comunicación preliminar). Estudios sobre la histeria. In: Obras completas de Sigmund Freud (Tomo I). Madrid: Biblioteca Nueva, 1973 [1893-5]. 
GARCÍA-CANCLINI, N. Diferentes, desiguales o desconectados. Revista CIDOB d'Afers Internacionals, v.66-67, p.113-33, 2004.

GRABOVSCHI, C.; LOIGNON, C.; FORTIN, M. Mapping the concept of vulnerability related to health care disparities: a scoping review. BMC Health Serv Res., v.13, p.94, 2013.

HAMMOND, D. The science of synthesis: exploring the social implications of General Systems Theory. Boulder: University of Colorado Press, 2003.

KOTHARI, M. L.; MEHTA, L. A. The trans-science aspects of disease and death. Perspect Biol Med., v.24, n.4, p.658-66, 1981.

KRIEGER, N. Theories for social epidemiology in the 21 st century: an ecosocial perspective. Int J Epidemiol., v.30, n.4, p.668-77, Aug 2001.

MORABIA, A. Reflexões históricas ao redor do livro Epidemiologia \& Saúde: Fundamentos, Métodos, Aplicações. Cadernos de Saúde Pública, v.29, n.6, p.1059$1062,2013$.

NAJMAN, J. M. Theories of disease causation and the concept of a general susceptibility: a review. Soc Sci Med Psychol Med Sociol., v.14, n.A(3), p.231-7, 1980.

NIERO M. Health, vulnerability and fragility: between determinants and dispositions. In: NIERO, M.; BERTIN, G. (Ed.) Vulnerability and social frailty: a theory of health inequalities. Roma: Franco Angeli, 2012. p.17-36.

PARKIN, R. T.; BALBUS, J. M. Variations in concepts of "susceptibility" in risk assessment. Risk Anal., v.20, n.5, p.603-12, 2000.

SAMAJA, J. Introducción a la epistemología dialéctica. Buenos Aires: Lugar Editorial, 1987.

- Epistemología y Metodología. Buenos Aires: Eudeba, 1993.

. Fundamentos epistemológicos de las ciências de la salud. Rio de Janeiro, 1997.

Tese (Dutorado) - Escola Nacional de Saúde Pública, Fundação Osvaldo Cruz.

A reprodução social e a saúde. Salvador: ISC/UFBA; Casa da Saúde, 2000.

. Desafios a la epidemiologia (pasos para uma epidemiologia 'Miltoniana'). Rev.

Bras. Epidemiol., v.6, p.2, p.105-20, 2003.

Epistemología de la Salud. Buenos Aires: Lugar Editorial, 2004.

SANTOS, B. S. A cruel pedagogia do vírus. São Paulo: Boitempo, 2020.

Conbecimento prudente para uma vida decente: Um discurso sobre as ciências revisitado. São Paulo: Cortez Editora, 2004.

SANTOS, M. A natureza do espaço. São Paulo: Hucitec; Edusp, 1996.

RESUMO - O objetivo deste texto é apresentar a pandemia Covid-19 como objeto complexo, com base numa proposta de modelagem heurística orientada por planos de ocorrência e interfaces hierárquicas. Para isso, em primeiro lugar, introduzo brevemente o pensamento de Juan Samaja, intelectual argentino representativo da epistemologia da 
complexidade. Em segundo lugar, apresento a teoria da holopatogênese, elaboração conceitual diretamente derivada da teoria samajiana das interfaces hierárquicas. Terceiro, aplico essa proposta de modelagem complexa para compreender a emergência da Covid-19, visando formular uma perspectiva integradora capaz de contribuir para o enfrentamento da pandemia. Finalmente, recorro à categoria de sobredeterminação para enriquecer esse esforço de compreensão teórica com um esquema organizador do conhecimento transdisciplinar sobre a pandemia Covid-19.

Palavras-CHave: Covid-19, Pandemia, Holopatogênese, Transdisciplinaridade, Sobredeterminação.

ABSTRACT - The aim of this text is to present the covid-19 pandemic as a complex object, based on a proposal of heuristic modeling guided by occurrence plans and hierarchical interfaces. For this, first of all, I briefly introduce the thoughts of Juan Samaja, Argentine scholar representative of the epistemology of complexity. Secondly, I present the holopathogenesis theory, a conceptual elaboration directly derived from Samaja's theory of hierarchical interfaces. Thirdly, I apply this proposal of complex modeling to understand the emergence of covid-19 as a problem, aiming to formulate an integrative perspective that might contribute to overcome the pandemic. Finally, I propose using the category of overdetermination to enrich this effort of theoretical understanding with an organizing scheme of transdisciplinary knowledge on the covid-19 pandemic.

KErWORDS: Covid-19, Pandemic, Holopathogenesis, Transdisciplinarity, Overdetermination.

Naomar de Almeida Filho é titular da Cátedra de Educação Básica da Universidade de São Paulo, parceria do Instituto de Estudos Avançados da USP e do Itaú Social, professor titular (aposentado) de Epidemiologia no Instituto de Saúde Coletiva da Universidade Federal da Bahia; pesquisador I-A do Conselho Nacional de Desenvolvimento Científico e Tecnológico (CNPq). @- naomaralmeida@gmail.com / https://orcid.org/0000-0002-4435-755X.

Recebido em 8.6.2020 e aceito em 26.6.2020.

I Instituto de Estudos Avançados, Universidade de São Paulo, São Paulo, Brasil. 
